\title{
Online Implementation of Inequality Constraints Monitoring in Dynamical Systems
}

\author{
Nadia Zanzouri, Ramzi Ben Messaoud, and Mekki Ksouri \\ Laboratory ACS, Department of Electrical Engineering, National Engineering School of Tunis, P. O. Box 37, \\ 1002 Tunis Belvedere, Tunisia \\ Correspondence should be addressed to Nadia Zanzouri, nadia.zanzouri@enit.rnu.tn
}

Received 13 September 2010; Revised 16 April 2011; Accepted 12 June 2011

Academic Editor: Ricardo Dunia

Copyright ( $\odot 2011$ Nadia Zanzouri et al. This is an open access article distributed under the Creative Commons Attribution License, which permits unrestricted use, distribution, and reproduction in any medium, provided the original work is properly cited.

This paper deals with fault detection in dynamical systems where the state variables evolutions are constrained by inequality constraints. The latter corresponds either to physical limitations or to safety specification. Two classical residual generation approaches are studied, namely, parity space and unknown input observer approaches, and are extended to monitor the inequality constraints. A practical implementation on a real process is performed and permits to validate the relevance of the proposed methods.

\section{Introduction}

Fault diagnosis is playing a crucial role in modern industrial processes to enhance the dependability of such systems. This has led to the development of a wide variety of modelbased approaches such as parity space, observer-based, or parameter identification methods $[1,2]$.

Dynamical systems are usually modelled by state and measurement equations that represent a set of equality constraints. In many practical applications, the state evolution is constrained by physical laws to stay in a given subspace. Moreover, it is generally required, for safety reasons, that the system works in particular conditions. As a consequence, a set of inequalities that constrain the evolution of the state variables in normal (no-fault) situation may be added to the state equations.

Monitoring a practical application requires not only the detection and isolation of the faults that corrupt the state evolution, but also the checking of the inequality constraints fulfilment. Even if the inequality constraints monitoring is very relevant for practical applications, it has not been extensively studied. The parity space approach has been extended in $[3,4]$, to this aim. The authors proposed to rewrite the inequalities as added equality constraints, thus to project the extended set of equality constraints in the so-called parity space. This leads to generating new residual signals that may be called inequality constraints residuals (IC-residuals), which should stay positive under a normal situation and become negative when inequalities are corrupted. It has been pointed out that faults that corrupt the inequality constraints do not necessarily corrupt the state equations and thus cannot be detected by classical residuals. In this paper, we came back first on the parity space approach and propose a substitution method to generating the IC-residuals. Then, we propose to use an unknown input observer whose objective is to detect internal and external faults. The conditions to design such full or reduced-order observer are established.

This paper is organised as follows: Section 2 briefly recalls the principle of the parity space approaches, and its extension to inequality constraints. In Section 3, we propose to design unknown input observers to estimate the inequality constraint residuals. In Section 4, a reducedorder observer is proposed. Finally, we show the results of a practical implementation of the two IC-residuals generation approaches on a real process. We conclude the paper with some remarks and orientations of our future work. 


\section{Preliminaries}

Consider a dynamic linear system described by

$$
\begin{aligned}
& \dot{x}(t)=A x(t)+B u(t)+F_{1} d(t), \\
& y(t)=C x(t)+D u(t)+F_{2} d(t),
\end{aligned}
$$

where $x(t) \in \mathfrak{R}^{n}, u(t) \in \mathfrak{R}^{r}$, and $y(t) \in \mathfrak{R}^{m}$ are the state, input, and output vectors, respectively. $d(t) \in \mathfrak{R}^{k}$ represents a fault signal. In the following sections, this kind of fault, which corrupts the state equations, will be qualified as internal fault. Matrices $A, B, C, D, F_{1}$, and $F_{2}$ are known constant matrices of appropriate dimensions.

The purpose of the parity space approach is to generate a residual that will inform us about the state of the studied system. The principle of this approach is to derive the output $y(t)$ up to order $s$, which leads to the following form:

$$
Y(t, s)-G_{s} U(t, s)=H_{s} x(t)+F_{s} D_{1}(t, s) .
$$

With

$$
\begin{aligned}
& Y(t, s)=\left(\begin{array}{c}
y \\
\dot{y} \\
\vdots \\
y^{(s)}
\end{array}\right), \quad U(t, s)=\left(\begin{array}{c}
u \\
\dot{u} \\
\vdots \\
u^{(s)}
\end{array}\right), \quad D_{1}(t, s)=\left(\begin{array}{c}
d \\
\dot{d} \\
\vdots \\
d^{(s)}
\end{array}\right) \text {, } \\
& H_{s}=\left(\begin{array}{c}
C \\
C A \\
\vdots \\
C A^{s}
\end{array}\right), \quad G_{s}=\left(\begin{array}{ccccc}
D & 0 & 0 & \cdots & 0 \\
C B & D & 0 & \cdots & 0 \\
C A B & C B & D & \cdots & 0 \\
\vdots & \vdots & \ddots & \ddots & \vdots \\
C A^{s-1} B & \cdots & C A B & C B & D
\end{array}\right) \text {, } \\
& F_{s}=\left(\begin{array}{ccccc}
F_{2} & 0 & 0 & \cdots & 0 \\
C F_{1} & F_{2} & 0 & \cdots & 0 \\
C A F_{1} & C F_{1} & F_{2} & \cdots & 0 \\
\vdots & \vdots & \ddots & \ddots & \vdots \\
C A^{s-1} F_{1} & \cdots & C A F_{1} & C F_{1} & F_{2}
\end{array}\right) .
\end{aligned}
$$

The unknown variable $x(t)$ (state vector) may be eliminated using a projection matrix $W[5]$ as follows:

$$
W H_{\mathrm{s}}=0 .
$$

The rows of $W$ define the so-called parity space.

The projection of (2) in the parity space leads to the computation form (5) and evaluation form (6) of the residual $r(t)$ :

$$
\begin{gathered}
r(t)=W\left(Y(t, s)-G_{s} U(t, s)\right), \\
r(t)=W F_{s} D_{1}(t, s) .
\end{gathered}
$$

The residual $r(t)$ is computed using online input and output values (5). $r(t)$ will be different from zero in the presence of an internal fault $d(t)$ and remains equal to zero in the normal $(d(t)=0)$ situation.

\section{Extension to Dynamical Systems Subject to Inequality Constraints}

In practical systems, the system states must stay in a given safe domain during a normal operation. Thus, a set of inequality constraints may be added as follows:

$$
H x(t) \leq h,
$$

where $h \in \mathfrak{R}^{q}$ is a constant vector (expresses the saturation level), $H$ is a real matrix of dimension $(q \cdot n)$, and $x(t)$ is a state vector of dimension $n$.

Define the following signal $v(t)$ as

$$
v(t)=-H x(t)+h,
$$

where $v(t) \in \mathfrak{R}^{q}$ is a real, nonnegative vector. It represents an indicator of the inequality constraint validity (7). $v(t)$ expresses the distance that separates the inequality constraints from their saturation level. The test diagnosis consists in checking the nonnegativity of the components of $v(t)$ (i.e., it exist elements $v_{i}(t)<0$ ), so the constraint $i$ is violated, then an alarm signal is generated as a result of a fault. Thus, $v(t)$ may be considered as an IC-residual.

3.1. Parity Space Approach. In [3, 4], the authors have shown how $v(t)$ may be computed using the parity space approach.

The constraint equality (8) is added to (2) to obtain

$$
\begin{gathered}
Y(t, s)-G_{s} U(t, s)=H_{s} x(t)+F_{s} D_{1}(t, s), \\
v(t)=-H x(t)+h .
\end{gathered}
$$

Consider the following projection matrix $W_{p}$ :

$$
W_{p}\left[\begin{array}{cc}
H_{s} & F_{s} \\
-H & 0
\end{array}\right]=0, \quad \text { with } W_{p}=\left[W_{p 1}, W_{p 2}\right]
$$

$W_{p 1}$ and $W_{p 2}$ have appropriate dimensions. Using this partitioning:

$$
\begin{gathered}
W_{p 1} H_{s}=W_{p 2} H, \\
W_{p 1} F_{s}=0 .
\end{gathered}
$$

Projecting (9a), (9b) using $W_{p}$ leads to

$$
W_{p 2} v(t)=-W_{p 1} Y(t, s)+W_{p 1} G_{s} U(t, s)+W_{p 2} h
$$

And one obtains

$$
v(t)=W_{p 2}^{+}\left(-W_{p 1} Y(t, s)+W_{p 1} G_{s} U(t, s)+W_{p 2} h\right),
$$

where $W_{p 2}^{+}$is the left pseudoinverse of $W_{p 2}$. So, $v(t)$ is expressed as a function of the known variables (inputs and outputs). 
3.1.1. Substitution Method Design. From (9a), we express the unknown state with the known input and output variables. First, we eliminate the vector $D_{1}(t, s)$ of the expression (9a) by a projection matrix $W_{s}$ such that

$$
W_{s} F_{s}=0
$$

$W_{s}$ exists if $m(s+1)>\operatorname{rank}\left[F_{s}\right]$. We obtain the following equation:

$$
W_{s}\left(Y(t, s)-G_{s} U(t, s)\right)=W_{s} H_{s} x(t) .
$$

It is assumed that $W_{s} H_{s}=\Omega$.

If $\Omega$ is regular matrix, then

$$
x(t)=\Omega^{-1} W_{s}\left(Y(t, s)-G_{s} U(t, s)\right)
$$

otherwise

$$
x(t)=\Omega^{+} W_{s}\left(Y(t, s)-G_{s} U(t, s)\right),
$$

where $\Omega^{+}=\left(\Omega^{T} \Omega\right)^{-1} \Omega^{T}$ a left pseudoinverse of $\Omega$.

Substituting $x(t)$ in $(9 \mathrm{~b})$,

$$
v(t)=-H \Omega^{+} W_{s}\left(Y(t, s)-G_{s} U(t, s)\right)+h .
$$

The advantage of the substitution approach is the evaluation form of the IC-residual $v(t)$ which is not possible to obtain by the projection method.

The procedure consists in eliminating the matrix $\left[I_{m(s+1)}-G_{s}\right]$ using a projection matrix $W_{d}$ as follows:

$$
\left[\begin{array}{ll}
I_{m(s+1)} & -G_{s}
\end{array}\right]\left[\begin{array}{c}
Y(t, s) \\
U(t, s)
\end{array}\right]=H_{s} x(t)+F_{s} D_{1}(t, s) .
$$

We obtain $x(t)=-\Omega_{d}^{+} W_{d} F_{s} D_{1}(t, s)$, where $\Omega_{d}=W_{d} H_{s}$.

By substituting $x(t)$ in the equation $v(t)$,

$$
v(t)=H \Omega_{d}^{+} W_{d} F_{s} D_{1}(t, s)+h .
$$

The fault vector $D_{1}(t, s)$ can be expressed with the inputs and outputs variables by eliminating the state vector with projection matrix $W$. From (9a), find $W$ such that $W H_{s}=0$ :

$$
W\left(Y(t, s)-G_{s} U(t, s)\right)=W F_{s} D_{1}(t, s)
$$

$W$ exists if $m(s+1)>\operatorname{rank}\left[H_{s}\right]$,

$$
D_{1}(t, s)=\Gamma^{+} W\left(Y(t, s)-G_{s} U(t, s)\right)
$$

$\Gamma=W F_{s}, \Gamma^{+}$is the left pseudoinverse of $\Gamma$.

3.2. Unknown Input Observer Approach. In the literature, many well-known results are available for the design of a state observer [6-10]. The simplicity of its design and the resolution of the difficulty imposed by missing measurements make the observer an attractive general design component [11]. We are interested here in designing an unknown input inequality constraint observer for linear systems subject to inequality constraints. Few works are interested in this problem and are not used in diagnosis. So, we propose an original design based on a constraint observer approach.

Consider a linear system described by

$$
\begin{gathered}
\dot{x}(t)=A x(t)+B u(t)+F d(t), \\
y(t)=C x(t), \\
v(t)=-H x(t)+h .
\end{gathered}
$$

It is assumed that the matrix $F$ is full column rank and that the pair $(A, C)$ is observable.

3.2.1. Constraint Observer Design. We propose an estimation of the IC-residual $v(t)$.

To determine $\hat{v}(t)$, we can estimate the state vector $x(t)$ as follows:

$$
\hat{v}(t)=-H \hat{x}(t)+h,
$$

$\hat{v}(t)$ and $\hat{x}(t)$ represent the estimates of $v(t)$ and $x(t)$, respectively.

The test diagnosis consists in checking the nonnegativity of vector $\hat{v}(t)$.

As it is shown above, we have estimated the entire state $x(t)$ to obtain $\hat{v}(t)$. This is not always necessary as sometimes there are constraints that do not use all the states. Therefore, we construct an observer that estimates directly the constraints. This observer is called constraint observer. It is written in the following form:

$$
\begin{gathered}
\dot{\omega}(t)=N \omega(t)+L y(t)+G u(t), \\
\hat{v}_{1}(t)=\omega(t)+E y(t) .
\end{gathered}
$$

We assume that $v_{1}(t)=v(t)-h$ and $\hat{v}_{1}(t)=\hat{v}(t)-h$.

$\omega(t)$ : state vector of observer and matrices $N, L, G$, and $E$ are to be designed so that $\hat{v}_{1}(t)$ asymptotically estimates $v_{1}(t)\left(\hat{v}_{1}(t) \rightarrow v_{1}(t)\right)$.

Let an error vector $e(t)$ be defined as

$$
e(t)=\hat{v}_{1}(t)-v_{1}(t)=\omega(t)+E y(t)+H x(t) .
$$

Hence,

$$
\begin{aligned}
\dot{e}(t)= & \dot{\hat{v}}_{1}(t)-\dot{v}_{1}(t)=\dot{\omega}(t)+E \dot{y}(t)+H \dot{x}(t) \\
= & (H+E C) \dot{x}(t)+\dot{\omega}(t) \\
= & (H+E C)(A x(t)+B u(t)+F d(t)) \\
& +N \omega(t)+G u(t)+L C x(t) .
\end{aligned}
$$

We assume

$$
P=H+E C .
$$

And replace $\omega(t)=e(t)-P x(t)$ into (27), so error dynamic $\dot{e}(t)$ is equal to

$$
\begin{aligned}
\dot{e}(t)= & N e(t)+(P A+L C-N P) x(t)+(P B+G) u(t) \\
& +P F d(t) .
\end{aligned}
$$


To obtain an error dynamic independent of $x(t), u(t)$, and $d(t)$ as the form, $\dot{e}(t)=N e(t)$ with $N$ being a Hurwitz (stability) matrix, the following conditions are to be satisfied:

$$
\begin{gathered}
N P=P A+L C, \\
P B+G=0, \\
P F=0, \\
P=H+E,
\end{gathered}
$$

$N$ is stable.

This is to guarantee the convergence of $e(t)(e(t) \rightarrow 0$ as $t \rightarrow \infty)$.

The well-known necessary and sufficient conditions [8] for the existence of this unknown input observer are

$$
\begin{gathered}
\operatorname{rank}[C F]=\operatorname{rank}[F], \\
\operatorname{Pair}((P A), C) \text { is observable. }
\end{gathered}
$$

Condition (31) is deduced from (30c) by replacing $P$ by its expression: $P=H+E C$. Condition (32) is deduced from (30a). A similar proof is given in detail in the next section.

If conditions (30a)-(30d), (31), and (32) are fulfilled, the matrix $N$ is found: $N=P A-K C$ with $K$ being a gain matrix chosen so that the pair $((P A), C)$ is observable. The gain $K$ can be chosen by a variety of optimisation or pole placement technique; see [12].

From (30a)-(30d), the matrix $L$ is determined:

$$
L=-K+N\left(P C^{+}-C^{+}\right) .
$$

3.2.2. Reduced-Order Constraint Observers. Now, we construct an observer which takes into account that a part of the state is given by system outputs and so it is already available by direct measurement. An observer of lower dimension called reduced-order observer [11] can be designed.

It can also be assumed that the matrix $C$ takes the form $C=\left[I_{m} 0\right] . C$ is partitioned into an $m * m$ identity matrix and $m *(n-m)$ zero matrix. The proposed reducedconstraint observer has a lower-order $q$, where $q \leq n-m$. Its design requires to decompose the matrices $A, F$, and $H$ as follows:

$$
A=\left[\begin{array}{ll}
A_{11} & A_{12} \\
A_{21} & A_{22}
\end{array}\right], \quad F=\left[\begin{array}{l}
F_{1} \\
F_{2}
\end{array}\right], \quad H=\left[\begin{array}{ll}
H_{1} & H_{2}
\end{array}\right]
$$

with

$$
\begin{gathered}
A_{11} \in \mathfrak{R}^{m * m}, \quad A_{12} \in \mathfrak{R}^{m *(n-m)}, \quad A_{21} \in \mathfrak{R}^{(n-m) * m}, \\
A_{22} \in \mathfrak{R}^{(n-m) *(n-m)}, \quad F_{1} \in \mathfrak{R}^{m * k}, \quad F_{2} \in \mathfrak{R}^{(n-m) * k}, \\
H_{1} \in \mathfrak{R}^{q * m}, \quad H_{2} \in \mathfrak{R}^{q *(n-m)} .
\end{gathered}
$$

By substituting these matrices in (30a)-(30d) and decomposing the matrix $P$ as follows: $P=\left[\begin{array}{ll}P_{1} & P_{2}\end{array}\right]$ with $P_{1} \in \mathfrak{R} q * m$ and $P_{2} \in \mathfrak{R}^{q *(n-m)}$, we obtain

$$
\begin{gathered}
L+P_{1} A_{11}+P_{2} A_{21}=N P_{1}, \\
N P_{2}=P_{1} A_{12}+P_{2} A_{22}, \\
P B=-G, \\
P_{1} F_{1}+P_{2} F_{2}=0, \\
H_{2}=P_{2}, \\
H_{1}+E=P_{1} .
\end{gathered}
$$

The necessary and sufficient conditions for the existence of such reduced observer are given by the following proposition:

$$
\begin{aligned}
& \operatorname{rank}\left[-H_{2} F_{2}\right]=\operatorname{rank}\left[F_{1}\right], \\
& \text { Pair }\left(K_{1}, K_{2}\right) \text { is observable. }
\end{aligned}
$$

Proof. From (36e) and (36d), we obtain

$$
P_{1} F_{1}=-H_{2} F_{2} .
$$

$P_{1}$ exists if $\operatorname{rank}\left[-H_{2} F_{2}\right]=\operatorname{rank}\left[F_{1}\right]$.

Now, we can write $P_{1}$ as follows [13]:

$$
P_{1}=-H_{2} F_{2} F_{1}^{+}+Z\left(I_{m}-F_{1} F_{1}^{+}\right),
$$

where $Z$ is an arbitrary matrix of appropriate dimension and $F_{1}^{+}$is the generalized inverse of $F_{1}\left(F_{1}^{+}=\left(F_{1}^{T} F_{1}\right)^{-1} F_{1}^{T}\right)$.

From (36b), we obtain

$$
N=P_{1} A_{12} H_{2}^{+}+H_{2} A_{22} H_{2}^{+},
$$

$\mathrm{H}_{2}^{+}$is the generalized inverse of $\mathrm{H}_{2}$. Substitute (40) into (41); it gives

$$
N=K_{1}-Z K_{2} .
$$

With

$$
\begin{gathered}
K_{1}=H_{2}\left(-F_{2} F_{1}^{+} A_{12}+A_{22}\right) H_{2}^{+}, \\
K_{2}=\left(F_{1} F_{1}^{+}-I_{m}\right) A_{12} H_{2}^{+} .
\end{gathered}
$$

Matrix $Z$ is chosen so that matrix $N$ is stable. This condition is fulfilled if the pair $\left(K_{1}, K_{2}\right)$ is observable $[8,10]$.

The matrices $G, L$, and $E$ are obtained from (36a)-(36f).

Remark 1. A continuous time model in state space form is used, but all the results are easily transcribed in discrete time.

\section{Application to Tank System}

Consider the tank system of laboratory ACS depicted in Figure 1 . The cylindrical tanks $R_{1}, R_{2}, R_{3}$, and $R_{s}$ are connecting by pipes. A pump $P$ (nova 180) distributes water from the tank $R_{s}$ in both reservoirs $R_{1}$ and $R_{3}$ through 


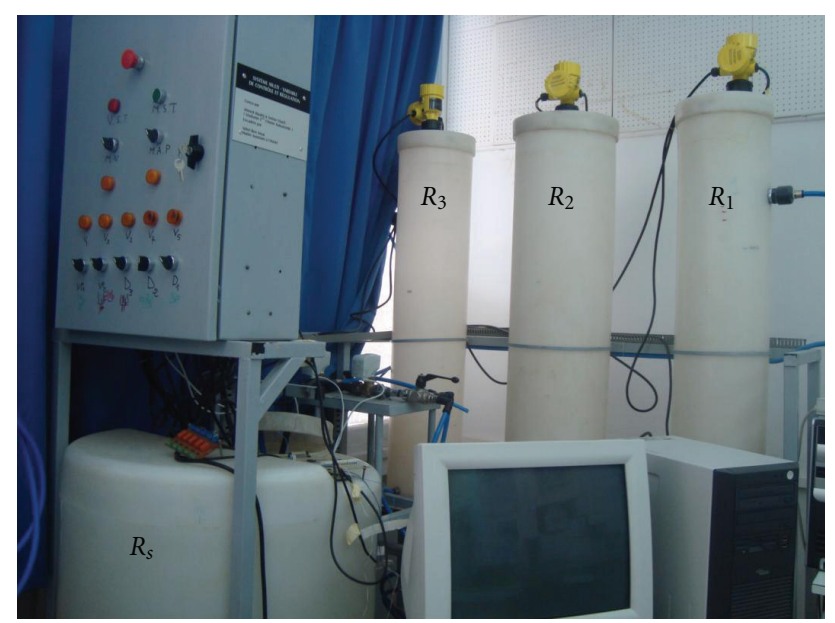

FIGURE 1: Tank system.

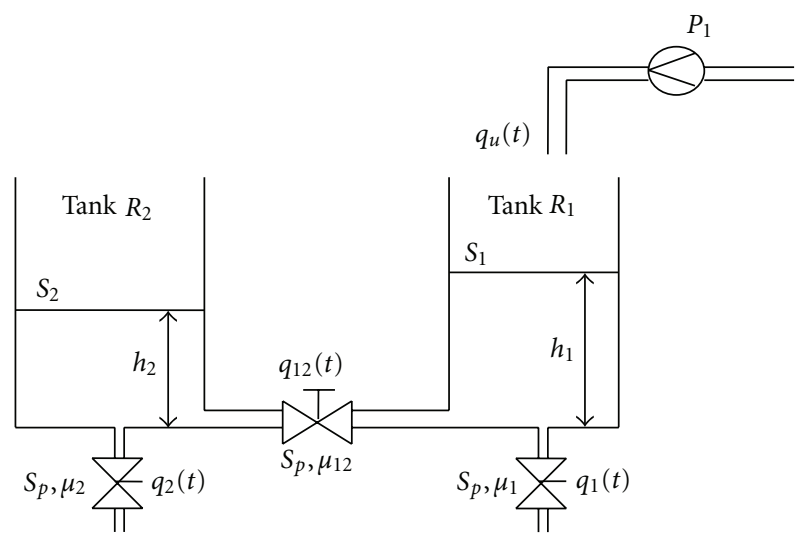

Figure 2: A simplified schematic diagram of a part tank process.

two electrovalves $V_{1}$ and $V_{2}$. Three level sensors (vega 61) measure the water levels $h_{i}$ of the three tanks.

Since there is a hardware problem with the valve $V_{23}$ connect $R_{2}$ to $R_{3}$, only the tanks $R_{1}$ and $R_{2}$ are used (Figure 2). The purpose of the tank system is to provide a continuous water flow $q(t)$ to a consumer.

The dynamic process of the water tank system can be illustrated by the model (44). It is written using the "mass balance" equations:

$$
\begin{gathered}
S_{1} \cdot \frac{d h_{1}(t)}{d t}=q_{u}(t)-q_{12}(t)-q_{1}(t), \\
S_{2} \cdot \frac{d h_{2}(t)}{d t}=q_{12}(t)-q_{2}(t), \\
y_{1}(t)=h_{1}(t), \\
y_{2}(t)=h_{2}(t),
\end{gathered}
$$

where

$$
q_{u}(t)=u(t)
$$

represents the pump flow.

$$
q_{i}(t)=\mu_{i} S_{p} \cdot \sqrt{2 \cdot g \cdot h_{i}(t)} \cdot(1-d(t)),
$$
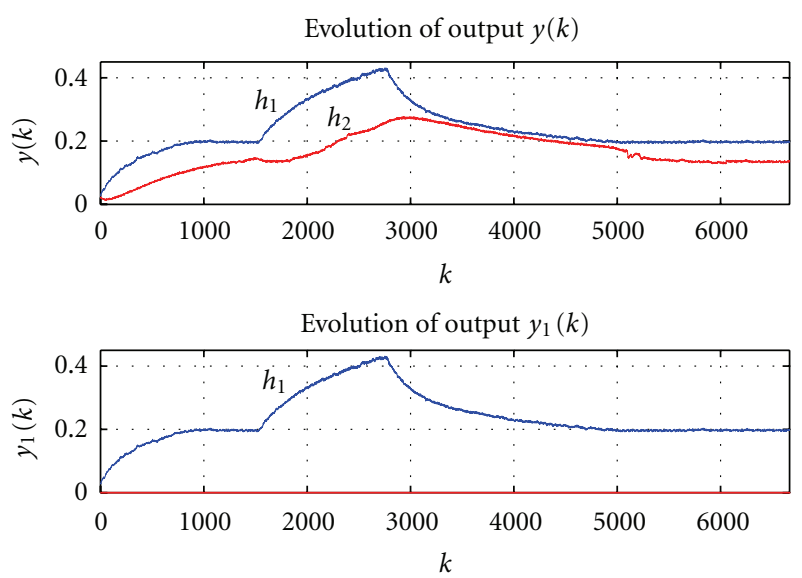

Figure 3: Water levels $h_{1}$ and $h_{2}$ in tanks $R_{1}$ and $R_{2}$.
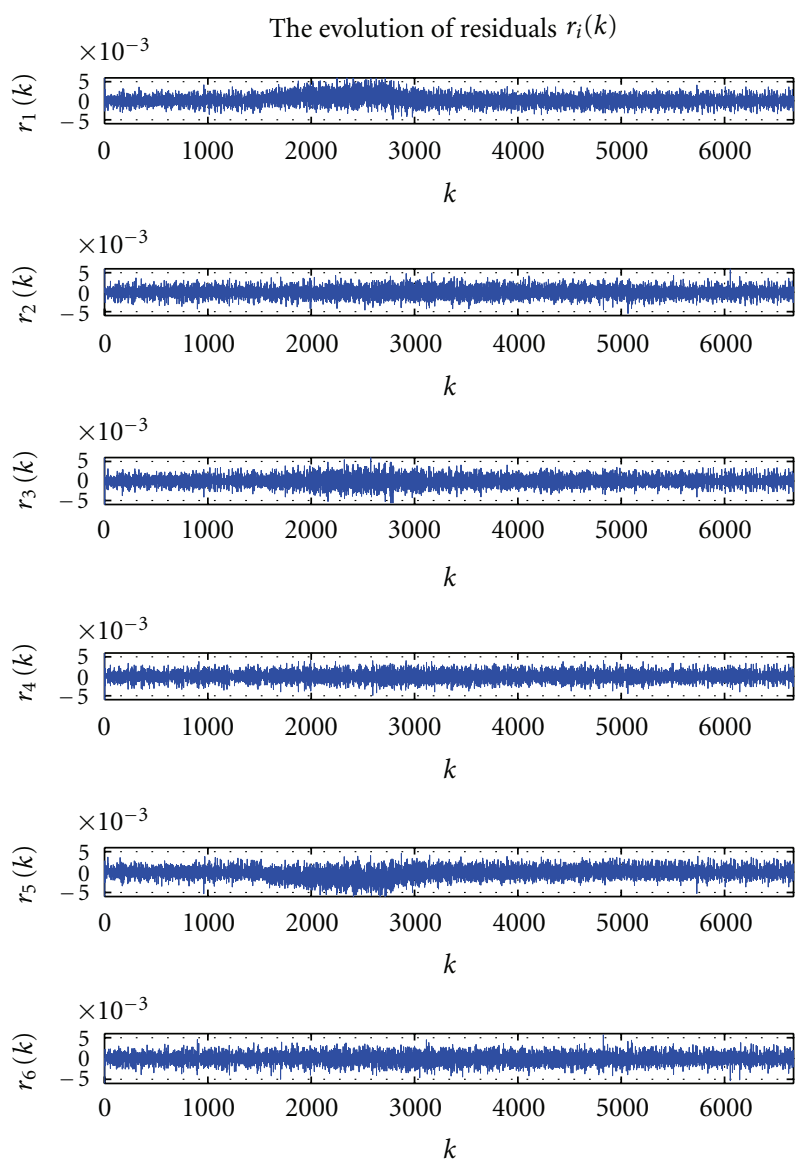

FIgURE 4: Residuals $r_{i}(k)$.

represents the water flow evacuated from the tank $R_{i}$ ( $i=$ $1,2), d(t)$ is the fault "clogging valve, $d(t)=1$ " otherwise 0 (healthy system), $q_{12}(t)$ represents the water flow between 
TABLE 1: Parameters of the tanks system.

\begin{tabular}{|c|c|c|}
\hline$S_{1}$ & $\begin{array}{l}\text { Cross-section area of the } \\
\text { tank } R_{1}\end{array}$ & $4.94 \times 10^{-2} \mathrm{~m}^{2}$ \\
\hline$S_{2}$ & $\begin{array}{l}\text { Cross-section area of the } \\
\text { tank } R_{2}\end{array}$ & $7.19 \times 10^{-2} \mathrm{~m}^{2}$ \\
\hline$S_{p}$ & $\begin{array}{l}\text { Cross-section area of the } \\
\text { valve that connects } R_{i}(i= \\
1,2) \text { to } R_{s}\end{array}$ & $8.89 \times 10^{-5} \mathrm{~m}^{2}$ \\
\hline$\mu_{1}$ & Constant coefficient & 0.2329 \\
\hline$\mu_{2}$ & Constant coefficient & 0.0599 \\
\hline$\mu_{12}$ & Constant coefficient & 0.0895 \\
\hline
\end{tabular}

tanks $R_{1}$ and $R_{2}$, which according to Torricelli's rule is given by

$$
q_{12}(t)=\mu_{12} S_{p} \cdot \operatorname{sign}\left(h_{1}(t)-h_{2}(t)\right) \sqrt{2 \cdot g \cdot\left|h_{1}(t)-h_{2}(t)\right|} .
$$

We additionally assume that the model of the system is not exactly known. Indeed, the output flow coefficients $\mu_{i}$ and $\mu_{12}$ are regarded as uncertain constant coefficients.

The values parameters of the tanks system are defined in Table 1.

The system (44) is linearised around its operating point and discretised for a sampling period $T=1 \mathrm{~s}$.

The water level in the supply tank $R_{1}$ and the middle tank $\mathrm{R}_{2}$ has to be maintained at a level:

$$
\begin{aligned}
& h_{1}<0.23 \mathrm{~m}, \\
& h_{2}<0.17 \mathrm{~m} .
\end{aligned}
$$

We get

$$
\begin{aligned}
& h(k+1)=\left[\begin{array}{ll}
0.9929 & 0.0025 \\
0.002 & 0.9971
\end{array}\right] h(k)+\left[\begin{array}{c}
20.2429 \\
0
\end{array}\right] u(k) \\
& +10^{-3}\left[\begin{array}{c}
0.8239 \\
0
\end{array}\right] d(k) \\
& y(k)=\left[\begin{array}{ll}
1 & 0 \\
0 & 1
\end{array}\right] h(k), \\
& v(k)=\left[\begin{array}{cc}
-1 & 0 \\
0 & -1
\end{array}\right] h(k)+\left[\begin{array}{l}
0.23 \\
0.17
\end{array}\right] \text {. }
\end{aligned}
$$

We consider a window observation $[k, k+2]$. Both substitution and projection methods lead to the same IC-residual $v_{s}(k)$. Thus, we have

$$
\begin{aligned}
v_{s}(k)= & -\left[\begin{array}{cccrcc}
1 & -0.013 & 0 & 0.0007 & 0 & 0.0007 \\
-0.0013 & 0.3353 & -0.0007 & 0.3343 & 0 & 0.3333
\end{array}\right] \\
& \times Y(k, 2)+10^{-17}\left[\begin{array}{rrr}
-0.0007 & -0.0003 & 0 \\
-0.5204 & -0.1486 & 0
\end{array}\right] U(k, 2) \\
& +\left[\begin{array}{c}
0.23 \\
0.17
\end{array}\right] .
\end{aligned}
$$

Using (5), we obtain the residual $r(k)$ :

$$
r(k)=\left(\begin{array}{cccccc}
0.6619 & 0.0016 & -0.3357 & 0.0010 & -0.3333 & 0.0003 \\
0.0016 & 0.6647 & 0.0007 & -0.3343 & -0.0003 & -0.3333 \\
-0.3357 & 0.0007 & 0.6667 & -0.0000 & -0.3310 & -0.0007 \\
0.0010 & -0.3343 & -0.0000 & 0.6667 & -0.0010 & -0.3324 \\
-0.3333 & -0.0003 & -0.3310 & -0.0010 & 0.6714 & -0.0016 \\
0.0003 & -0.3333 & -0.0007 & -0.3324 & -0.0016 & 0.6686
\end{array}\right) Y(k, 2)-\left(\begin{array}{cccc}
-13.4946 & -6.7471 & 0 \\
-0.0064 & -0.0063 & 0 \\
6.8437 & -6.6994 & 0 \\
-0.0331 & -0.0197 & 0 \\
6.7952 & 13.5908 & 0 \\
-0.0195 & -0.0329 & 0
\end{array}\right)(k, 2)
$$

Now, we assume that the level sensor of tank $R_{2}$ is broken, so the only water level $h_{1}$ in the tank $R_{1}$ is measured. Consequently, the output variable is written in the following form:

$$
y(k)=\left[\begin{array}{cc}
1 & 0
\end{array}\right] h(k) ; \quad C_{1}=\left[\begin{array}{ll}
1 & 0
\end{array}\right] .
$$

Using (30a)-(30d), the constraint observer system is

$$
\begin{aligned}
\omega(k+1)= & {\left[\begin{array}{cc}
-0.3 & 0 \\
0 & 0.9971
\end{array}\right] \omega(k)+\left[\begin{array}{cc}
0 & 0 \\
-0.002 & 0
\end{array}\right] y(k), } \\
& \hat{v}_{1}(k)=\omega(k)+\left[\begin{array}{cc}
-1 & 0 \\
0 & 0
\end{array}\right] y(k)
\end{aligned}
$$

and the existence conditions of the observer are fulfilled:

$$
\operatorname{rank}\left[\begin{array}{cc}
C_{1} & F
\end{array}\right]=\operatorname{rank}[F]=1 .
$$

The pair $((P A), C 1)$ is observable. It can be tested by using the smith form $[14,15]$ :

$$
\operatorname{rank}\left[\begin{array}{c}
z I_{2}-P A \\
C_{1}
\end{array}\right]=2,
$$

where $z$ is the operator variable of $z$-transform.

Now, a reduced-order observer is designed. The purpose is to estimate only the nonmeasurable constraint that 

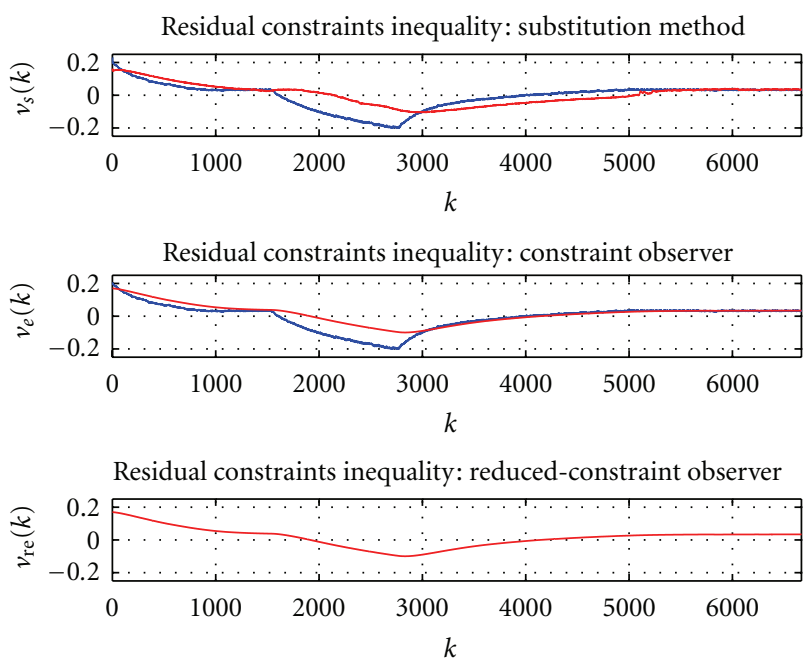

FIGURE 5: IC-residual $v(k)$.
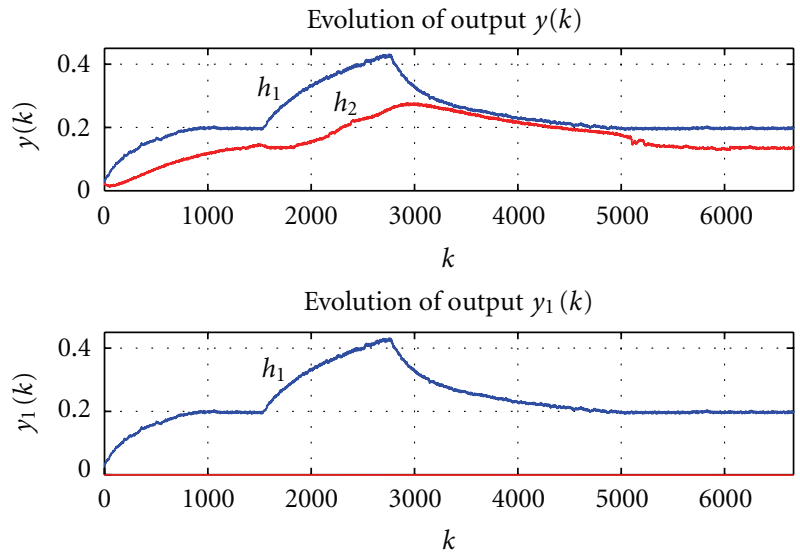

Figure 6: Water levels $h_{1}$ and $h_{2}$ in tanks $R_{1}$ and $R_{2}$.

monitors the water level in the second tank $R_{2}$. It requires to decompose the matrices $H, P, A$, and $F$ as follows:

$$
\begin{gathered}
H=\left[\begin{array}{ll}
H_{1} & H_{2}
\end{array}\right]=\left[\begin{array}{ll}
0 & -1
\end{array}\right], \quad F=\left[\begin{array}{l}
F_{1} \\
F_{2}
\end{array}\right]=10^{-3}\left[\begin{array}{c}
0,8239 \\
0
\end{array}\right], \\
A=\left[\begin{array}{ll}
A_{11} & A_{12} \\
A_{21} & A_{22}
\end{array}\right]=\left[\begin{array}{cc}
0.9929 & 0.0025 \\
0.002 & 0.9971
\end{array}\right] .
\end{gathered}
$$

By applying (36a)-(36f), the matrices of the observer are

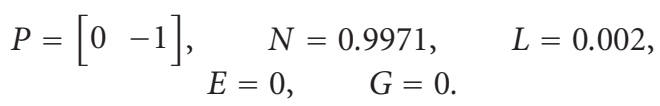

So, the dynamical observer is

$$
\begin{gathered}
\omega(k+1)=0.9971 \omega(k)+0.002 y(k), \\
\hat{v}_{1}(k)=\omega(k) .
\end{gathered}
$$

4.1. Experimental Results. The monitoring algorithms are implemented on the real process described in Figure 1. Two kinds of faults are introduced in the process:

(i) Fault 1: clogging of the output valve,

(ii) Fault 2: pump flow.

4.1.1. Fault 1. For the flow pump $q_{u}(t)=4.94 \times 10^{-5} \mathrm{~m}^{3} \cdot \mathrm{s}^{-1}$, the system stabilizes to water level $h_{1}=0.197 \mathrm{~m}$ for the tank $R_{1}$ and $h_{2}=0.136 \mathrm{~m}$ for the tank $R_{2}$. The clogging of the output valve between time $1530 \mathrm{~s}$ and $2761 \mathrm{~s}$ increases the water levels $h_{1}$ and $h_{2}$ and shows in Figure 3 an abnormal situation.

Also, the equality residuals $r_{i}(k)$ become sensitive to this fault. Figure 4 shows that the residuals are disturbed between time $1530 \mathrm{~s}$ and $2761 \mathrm{~s}$. Since they are noisy, an analysis signal technique must be used to improve the fault diagnosis [16].

It is seen that the IC-residuals $v(k)$ (Figure 5 ) generated by parity and observer approaches become negative in the time window [1530s, $2761 \mathrm{~s}]$. Hence, the inequality constraints are violated. Thus, the fault is detected. 

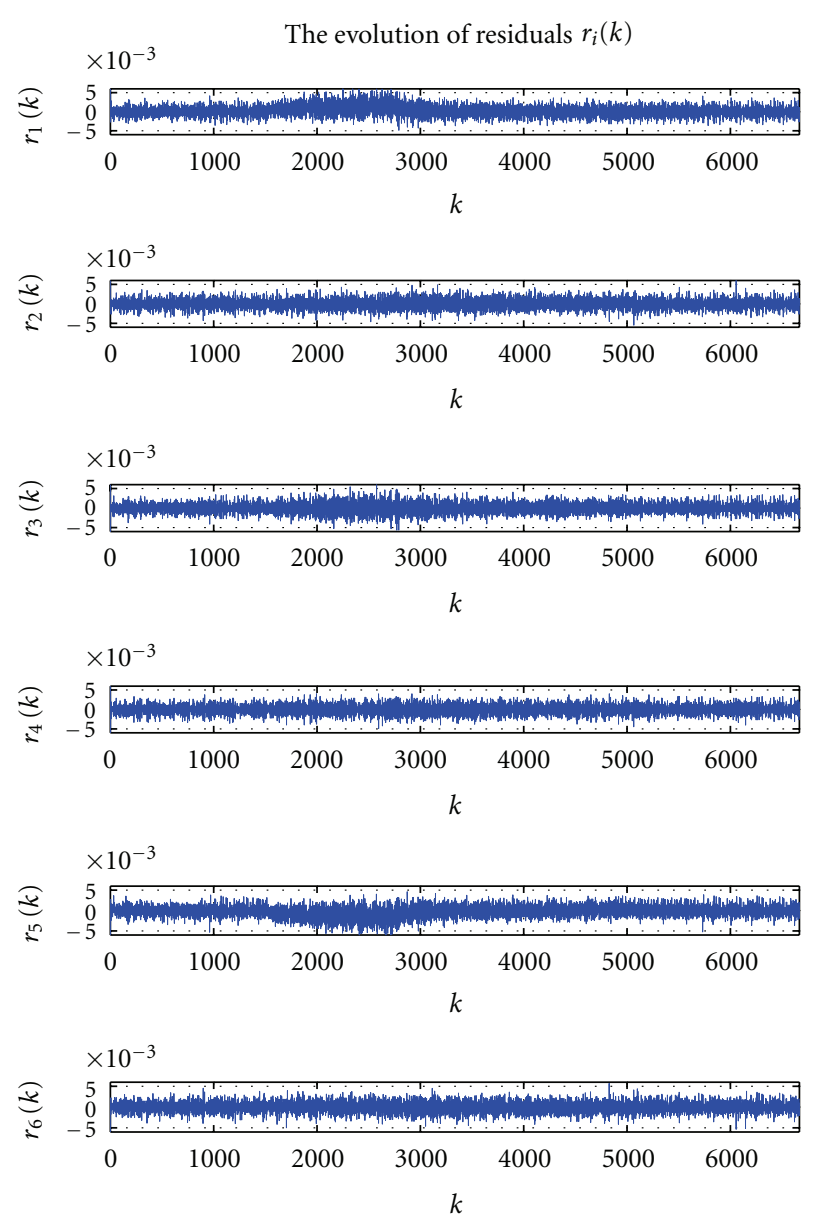

FIGURE 7: Residuals $r_{i}(k)$.

4.1.2. Fault 2. In normal operation, the flow pump is $q_{u}(k)=$ $4.94 \times 10^{-5} \mathrm{~m}^{3} \cdot \mathrm{s}^{-1}$. We have introduced a fault in the pump; it consists in increasing its flow $q_{u 1}(k)=1.027 \times 10^{-4} \mathrm{~m}^{3} \cdot \mathrm{s}^{-1}$ between time $1705 \mathrm{~s}$ and $2382 \mathrm{~s}$. The water levels $h_{1}$ and $h_{2}$ increase and show in Figure 6 an abnormal situation.

We remark (Figure 7) that the equality residuals are not very sensitive to this kind of fault. An analysis signal technique must be used to provide a good decision.

However, Figure 8 shows that the IC-residuals $v(k)$ become negative. This implies the violation of the inequality constraints which are due to the presence of a fault. So, the IC-residuals provide a good fault detection.

\section{Conclusion}

In this paper, the advantages provided by the IC-residual to fault detection have been shown. A substitution parity space technique has been proposed in order to express the IC-residual in the evaluation form. Full and reduced-order unknown input constraint observers are designed for the monitoring system. The obtained results are encouraging and similar to those obtained by parity space approach. It has been noted that the IC-residual detects the external faults not considered in the system model. An online implementation
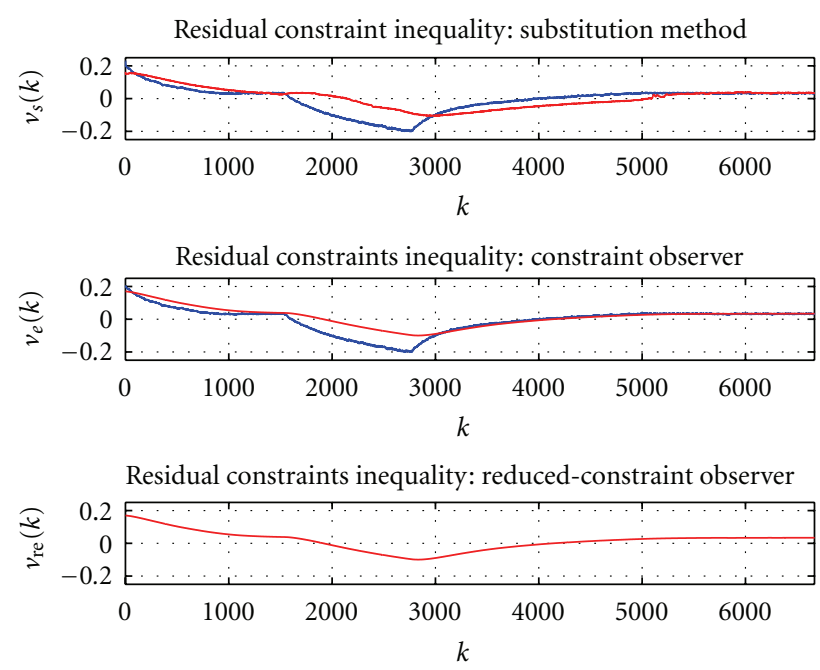

Figure 8: IC-residual $v(k)$.

of IC-residual on a real process is performed and permits to validate the relevance of the proposed methods.

Our future work will involve the improvement of the fault diagnosis and the fault tolerant control by reducing the fault detection delay.

\section{Acknowledgments}

The authors would like to thank Professor Vincent Cocquempot at the University of Lille for the discussion and helpful comments. They also thank the valuable comments and suggestions received from the reviewers of this paper.

\section{References}

[1] R. Isermann, "Process fault detection based on modeling and estimation methods-A survey," Automatica, vol. 20, no. 4, pp. 387-404, 1984.

[2] R. J. Patton, P. M. Frank, and R. N. Clark, Fault Diagnosis in Dynamical Systems, Theory and Application, Prentice Hall, 1989.

[3] M. Staroswiecki and D. Guerchouh, "A parity space approach for monitoring inequality constraints-part 1: static case," in Proceedings of 14th IFAC World Congress, pp. 109-114, Beijing, China, 1999.

[4] M. Staroswiecki and D. Guerchouh, "A parity space approach for monitoring inequality constraints_-part 2: dynamic case," in Proceedings of 14th IFAC World Congress, pp. 115-120, Beijing, China, 1999.

[5] E. Y. Chow and A. S. Willsky, "Analytical redundancy and the design of robust failure detection systems," IEEE Transactions on Automatic Control, vol. 29, no. 7, pp. 603-614, 1984.

[6] M. Hou and P. C. Müller, "Design of observers for linear systems with unknown inputs," IEEE Transactions on Automatic Control, vol. 37, no. 6, pp. 871-875, 1992.

[7] S. K. Chang, W. T. You, and P. L. Hsu, "General-structured unknown input observers," in Proceedings of the American Control Conference, pp. 666-670, Baltimore, Md, USA, July 1994. 
[8] M. Darouach, M. Zasadzinski, and S. J. Xu, "Full-order observers for linear systems with unknown inputs," IEEE Transactions on Automatic Control, vol. 39, no. 3, pp. 606-609, 1994.

[9] C. C. Tsui, "A new design approach to unknown input observers," IEEE Transactions on Automatic Control, vol. 41, no. 3, pp. 464-468, 1996.

[10] H. Trinh, T. Fernando, and S. Nahavandi, "Design of reducedorder functional observers for linear systems with unknown inputs," Asian Journal of Control, vol. 6, no. 4, pp. 514-520, 2004.

[11] D. G. Luenberger, "Introduction to observers," IEEE Transactions on Automatic Control, vol. 16, no. 6, pp. 596-602, 1971.

[12] A. E. Bryson Jr. and Y. C. Ho, Applied Optimal Control, Blasdell, Waltham, Mass, USA, 1969.

[13] R. M. Pringler and A. Rayner, A Generalized Inverse Matrices, Griffin, London, UK, 1971.

[14] H. Rosenbrock, State-Space and Multivariable Theory, Nelson, 1970.

[15] P. Kudva, N. Viswanadham, and A. Ramakrishna, "Observers for linear systems with unknown inputs," IEEE Transactions on Automatic Control, vol. 25, no. 1, pp. 113-115, 1980.

[16] S. Lesecq and S. Gentil, "Signal-based diagnostic algorithms integrating model validity in the decision," Control Engineering Practice, vol. 16, no. 9, pp. 1120-1131, 2008. 

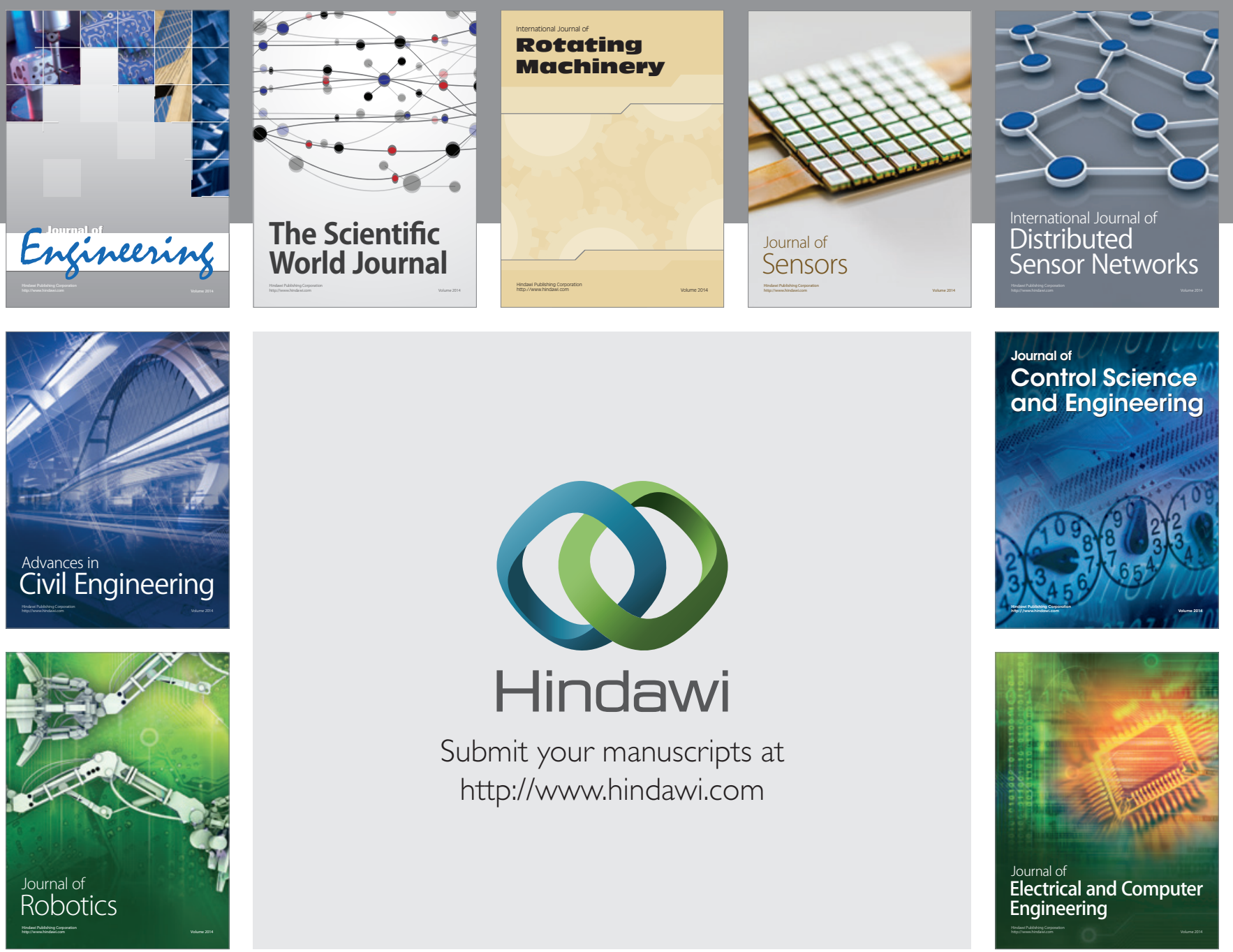

Submit your manuscripts at

http://www.hindawi.com
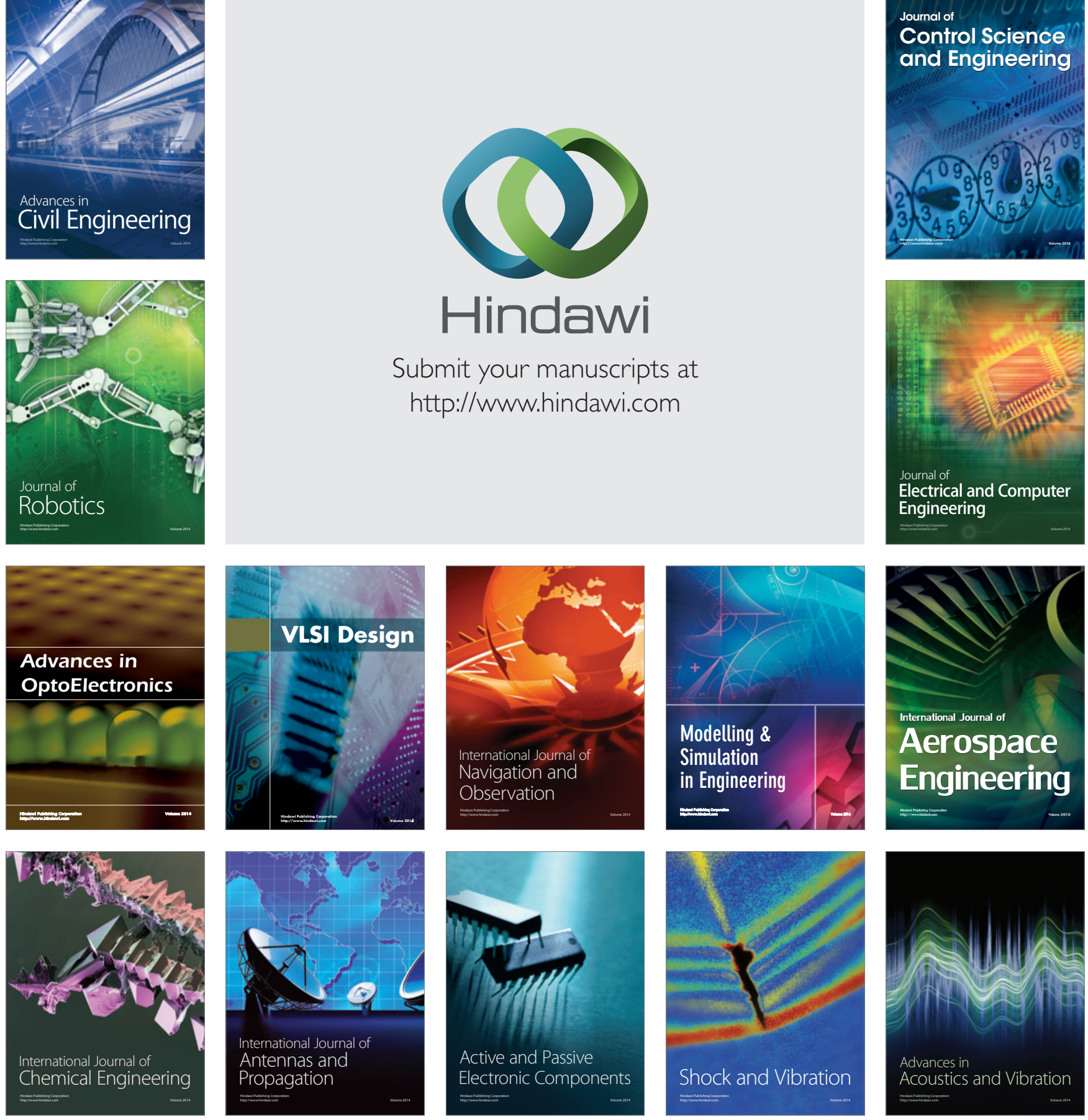